\title{
Counting Electrons Transferred through a Thin Alumina Film into Au Chains
}

\author{
N. Nilius, ${ }^{1, *}$ M. V. Ganduglia-Pirovano, ${ }^{2, *}$ V. Brázdová, ${ }^{2, \dagger}$ M. Kulawik, ${ }^{1}$ J. Sauer, $^{2}$ and H.-J. Freund ${ }^{1}$ \\ ${ }^{1}$ Fritz-Haber-Institut der MPG, Faradayweg 4-6, Berlin, Germany \\ ${ }^{2}$ Humboldt Universität zu Berlin, Institut für Chemie, Brook-Taylor-Str. 2, Berlin, Germany
}

(Received 30 July 2007; published 7 March 2008)

\begin{abstract}
Low-temperature STM measurements combined with density functional theory calculations are employed to study the adsorption of gold on alumina/ $\mathrm{NiAl}(110)$. The binding of $\mathrm{Au}$ monomers involves breaking of an oxide Al-O bond below the adatom and stabilizing the hence undercoordinated $\mathrm{O}$ ion by forming a new bond to an $\mathrm{Al}$ atom in the $\mathrm{NiAl}$. The adsorption implies negative charging of the adatom. The linear arrangement of favorable binding sites induces the self-organization of Au atoms into chains. For every ad-chain, the number of transfer electrons from the support is determined by analyzing the node structure of the corresponding highest occupied molecular orbital.
\end{abstract}

PACS numbers: 73.21.Fg, 68.37.Ef, 68.47.Jn, 71.15.Mb

Recent experiments demonstrated the importance of charge transfer processes through thin oxide films grown on metal supports [1]. The charge flow is triggered by an oxide-induced work function decrease in combination with the presence of electronegative adsorbates [2]. For example, negative charging of $\mathrm{Au}$ atoms on thin $\mathrm{MgO}$ films on $\mathrm{Ag}(001)$ has been deduced from the hexagonal arrangement of the adatoms and the formation of $2 \mathrm{D}$ islands at higher coverage $[1,3]$. Also the enhanced chemical reactivity of thin film systems has been traced back to charge transfer processes involving the metal support [4]. The possibility to control the charge state of adsorbates therefore enables a modification of the catalytic performance of thin film systems [5]. The investigation of charge transfer is, however, difficult from an experimental point of view and often relies on the evaluation of macroscopic surface properties, e.g., the work function [6] or the vibrational characteristic of probe molecules $[7,8]$. On the level of single adsorbates, charge exchange is mainly deduced from indirect information, such as the blocked aggregation of charged species or the appearance of sombrero shapes around adatoms in the STM [1,9].

In this work, we demonstrate the possibility to quantify charge transfer directly from the electron filling of quantum well states (QWS) that form in self-assembled Au chains on a thin alumina film on $\mathrm{NiAl}(110)$. These chains of 5-22 $\AA$ length have been observed in recent STM experiments [10]; however, neither their atomic configuration nor the driving force for a linear atom arrangement was derived from that study. DFT calculations and STM spectroscopy data presented here provide a detailed picture of the specific Au interaction with the oxide film and the $\mathrm{NiAl}$ below, and in particular, on the role of charge transfer into the ad-chains.

The alumina film is prepared by exposing $\mathrm{NiAl}(110)$ to $1200 \mathrm{~L}$ of $\mathrm{O}_{2}$ at $580 \mathrm{~K}$, followed by annealing to $1000 \mathrm{~K}$ [5]. Single Au atoms are deposited from a gold-coated tungsten filament onto the sample held at $10 \mathrm{~K}$ [Fig. 1(a)]. Because of their thermal energy when imping- ing the surface, the atoms have transient mobility to explore the potential landscape for adsorption and observed binding configurations are therefore equilibrium structures. The Au aggregates are imaged with an UHV-STM operated at $5 \mathrm{~K}$ [11]. Their electronic structure is investigated by differential conductance $(d I / d V)$ spectroscopy, performed with lock-in technique. Spin-polarized DFT calculations are carried out with the gradient-corrected PW91 exchange and correlation functional and a planewave basis set (cutoff $250 \mathrm{eV}$ ), as implemented in the VASP code [12]. The computational cell covers two elementary cells $\left(10.55 \times 17.89 \AA^{2}\right)$ of alumina on $\operatorname{NiAl}(110)$ and contains $80 \mathrm{Al}$ and $104 \mathrm{O}$ atoms in the oxide and 66



FIG. 1 (color online). (a) STM image of Au monomers and small aggregates on alumina/ $\mathrm{NiAl}(110)\left(U_{\text {sample }}=-1.5 \mathrm{~V}, I=\right.$ $0.5 \mathrm{nA}, 35 \times 35 \mathrm{~nm}^{2}$ ). (b) Model of the system used for DFT calculations. The white rectangle and the green parallelogram mark the alumina unit cell and the computational cell, respectively. The yellow circles denote $\mathrm{Au}$ atoms in the most stable dimer configuration. (c) Ball-stick model of monomer, trimer, and tetramer. The dashed lines indicate the broken oxide bonds. $\mathrm{Au}$ atoms in special binding sites are marked by connecting lines to the oxide film. 
NiAl unit cells [Fig. 1(b)]. To compensate the incommensurability between oxide and NiAl lattice constants along the [001] direction, the oxide unit cell is slightly expanded in this direction [13]. The Brillouin zone is sampled at the $\Gamma$ point. A systematic underestimation of band gaps by gradient-corrected functionals, (theory $4.2 \mathrm{eV}$ versus experiment $6.7 \mathrm{eV}$ for alumina/NiAl) allows only a qualitative comparison between peak positions in the calculated LDOS and the $d I / d V$ spectra.

The structure of the alumina film on $\mathrm{NiAl}(110)$ was recently identified by a combined STM/DFT study [13]. The film consists of (i) a topmost oxygen layer $\left(\mathrm{O}_{\text {top }}\right)$ with atoms in triangular and square arrangements, (ii) a hexagonal layer of fourfold and fivefold coordinated $\mathrm{Al}$ atoms $\left(\mathrm{Al}_{\text {top }}\right)$, (iii) a structurally identical $\mathrm{O}$ layer $\left(\mathrm{O}_{\text {inter }}\right)$ and (iv) an interfacial $\mathrm{Al}$ layer with atoms arranged in pentagons and heptagons $\left(\mathrm{Al}_{\text {inter }}\right)$. The distinct symmetry of the interfacial layer allows maximum overlap between the $\mathrm{Al}_{\text {inter }}$ atoms and the [001] oriented $\mathrm{Ni}$ rows of the $\mathrm{NiAl}$.

To identify preferential adsorption sites on the alumina, the binding energy of single Au atoms is calculated with respect to a gas-phase atom. No stable adsorption is revealed on $\mathrm{O}_{\text {top }}$ sites, whereas occupation of $\mathrm{Al}_{\text {top }}$ sites leads to strong binding, in particular, at those sites that are located above an $\mathrm{Al}$ atom in the $\mathrm{NiAl}$ support. Formation of an $\mathrm{Au}-\mathrm{Al}_{\text {top }}$ bond lifts the $\mathrm{Al}_{\text {top }}$ ion above the surface plane and increases the distance to the $\mathrm{O}_{\text {inter }}$ species below from 1.8 to $4.4 \AA$. As a result, this oxide bond breaks and a new bond forms between the $\mathrm{O}_{\text {inter }}$ and the $\mathrm{Al}$ atom of the support [Fig. 1(c)] [14]. In a simplified ionic picture, the bond rearrangement involves transfer of one electron from the $\mathrm{Al}_{\text {top }}$ to the $\mathrm{Au}$ (instead of to the $\mathrm{O}_{\text {inter }}$ below) and refilling of the $\mathrm{O}_{\text {inter }}$ states with a NiAl electron. Consequently, the coordination and electron number of $\mathrm{Al}_{\text {top }}$ and $\mathrm{O}_{\text {inter }}$ species do not change, while the $\mathrm{Au}$ atom becomes negatively charged due to electron transfer from the support [15]. Only ten of the $26 \mathrm{Al}_{\text {top }}$ ions in the oxide unit cell allow for this particular binding configuration (referred to as special sites, Fig. 1(b)]. Seven of them are fourfold coordinated $\mathrm{Al}_{\text {top }}$ ions with $1.53-2.03 \mathrm{eV}$ binding energy and three are fivefold coordinated sites that bind the $\mathrm{Au}$ with $1.41-1.57 \mathrm{eV}$. The electronic structure of Au adatoms is characterized by an $s-d$ like HOMO at $-1.3 \mathrm{eV}$, which mainly derives from the singly occupied $6 s$ orbital of the gas-phase atom [Fig. 2(a)]. The complete filling of this state reflects the additional electron at the $\mathrm{Au}_{1}$, as revealed also by a Bader-type analysis (Table I) and the vanishing spin density. The LUMO of the adatom has mainly $p_{\perp}$ character and is located at $+2.5 \mathrm{eV}$ above $E_{F}$.

The most stable dimer identified by DFT consists of two $\mathrm{Au}$ atoms placed on adjacent special $\mathrm{Al}_{\text {top }}$ sites and has an Au-Au binding length of $2.6 \AA$ [Fig. 1(b)]. Bond breaking in the alumina and restabilization via the $\mathrm{NiAl}$ takes place for both $\mathrm{Au}$ atoms and is accompanied by the transfer of two electrons into the dimer (Table I). The HOMO and HOMO-1 arise from the superposition of the $s-d_{z^{2}}$ orbitals
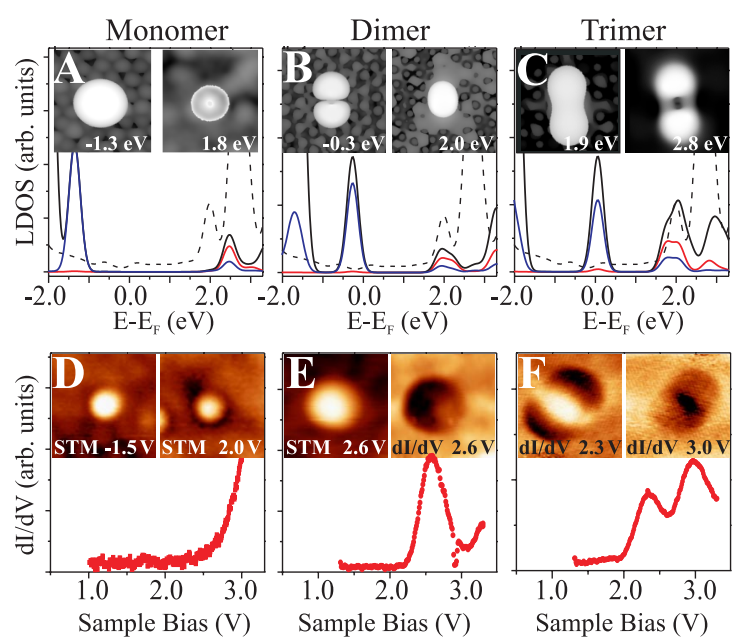

FIG. 2 (color online). (a) Spin-averaged LDOS and orbital shapes for the most stable monomer, (b) dimer and (c) trimer on alumina/NiAl. The black line marks the total Au LDOS, the blue and red lines denote the $s-d_{z^{2}}$ and $p_{\perp}$ contributions, respectively. The dashed line depicts the alumina LDOS. (d) Experimental $d I / d V$ spectrum and topographic images of an $\mathrm{Au}$ monomer taken at two different voltages. (e) $d I / d V$ spectrum of a dimer with corresponding topographic and $d I / d V$ image. (f) Trimer spectrum with $d I / d V$ images taken at the two peak positions [17]. All images are $4.5 \times 4.5 \mathrm{~nm}^{2}$ in size; the set-point for spectroscopy was set with $3.0 \mathrm{~V}$.

of the adatoms and show one and zero nodes along the dimer axis ( $z$ axis), respectively [Fig. 2(b)]. Both states are well below $E_{F}$ and filled with the two initial Au $6 s$ electrons and two transfer-electrons from the NiAl. The LUMO at $+2.0 \mathrm{eV}$ results from a symmetric combination of the two Au $p_{\perp}$ orbitals and exhibits a slightly elongated orbital shape. The most stable trimer forms on three [001] orientated $\mathrm{Al}_{\text {top }}$ neighbors. Whereas the outer trimer atoms bind to the oxide via the bond-breaking mechanism, the central one is only stabilized by the Au-Au interaction [Fig. 1(b)]. Three $s-d_{z^{2}}$ states with zero, one and two nodal planes emerge in the trimer LDOS. They are occupied by five electrons, three from the initial $\mathrm{Au} 6 s^{1}$ orbitals and two being transferred from the NiAl (Table I). The singly occupied $\mathrm{HOMO}$ at $E_{F}$ is split by $0.25 \mathrm{eV}$ into a spin-up and a spin-down state, which leads to a magnetic ground state of the $\mathrm{Au}_{3}$ with one $\mu_{\text {Bohr magnetic moment. The }}$ unoccupied LDOS consists of symmetric/antisymmetric combinations of the $p_{\perp}$ orbitals, giving rise to states at 1.9 and $2.8 \mathrm{eV}$. Whereas the lower state shows elliptical symmetry, the higher one has a nodal plane in the center of the trimer [Fig. 2(c)].

Based on their DFT signature, small Au aggregates are identified in the experiment. Monomers appear as round protrusions in filled-state images (negative sample bias), but change into shallow maxima and even depressions at positive bias [Fig. 2(d)]. This contrast reversal reflects the absence of states between $E_{F}$ and the alumina conduction band, which renders tunneling into the $\mathrm{Au}_{1}$ species inefficient. The sombrero appearing around $\mathrm{Au}$ monomers at 
TABLE I. Binding parameters for Au aggregates on alumina/NiAl(110).

\begin{tabular}{|c|c|c|c|c|c|c|c|}
\hline & \multicolumn{5}{|c|}{ One dimensional (1D) chains } & \multicolumn{2}{|c|}{ 2D islands } \\
\hline & $\begin{array}{c}\text { Broken } \\
\text { alumina } \\
\text { bonds }\end{array}$ & $\begin{array}{l}\text { Transferred } \\
\text { charges } \\
\text { from Nial }\end{array}$ & $\begin{array}{l}\text { Quantum } \\
\text { number } n \\
\text { of HOMO }\end{array}$ & $\begin{array}{c}\text { Number of } \\
\text { electrons in } \\
\text { all QWS }\end{array}$ & $\begin{array}{l}\text { Magnetic } \\
\text { moment in } \\
\mathrm{Au} / \mathrm{NiAl}^{\mathrm{b}}\end{array}$ & $\begin{array}{c}\text { Broken } \\
\text { alumina } \\
\text { bonds }\end{array}$ & $\begin{array}{c}\text { Transferred } \\
\text { charges from } \\
\mathrm{NiAl}^{\mathrm{a}}\end{array}$ \\
\hline Monomer & 1 & -0.95 & 1 & 2 & $0 / 1$ & \multirow{3}{*}{\multicolumn{2}{|c|}{ Unstable }} \\
\hline Dimer & 2 & -1.88 & 2 & 4 & $0 / 0$ & & \\
\hline Trimer & 2 & -1.71 & 3 & $5^{\mathrm{c}}$ & $1 / 0$ & & \\
\hline Tetramer & 2 & -1.91 & 3 & 6 & $0 / 0$ & 2 & -1.87 \\
\hline Pentamer & 3 & -2.86 & 4 & 8 & $0 / 1$ & 2 & -1.54 \\
\hline Hexamer & 3 & -3.12 & 5 & $9^{c}$ & $1 / 1$ & 2 & -1.89 \\
\hline Heptamer & 3 & -2.88 & 5 & 10 & $0 / 1$ & 2 & -1.45 \\
\hline
\end{tabular}

${ }^{\mathrm{a}}$ As derived from Bader charge analysis, [e].

${ }^{b} \mathrm{NiAl}$ magnetic moment due to hole remaining after charge transfer into ad-chains, $\left[\mu_{B}\right]$.

${ }^{\mathrm{c}} \mathrm{HOMO}$ located at the Fermi level and only singly occupied.

2.0-3.0 $\mathrm{V}$ provides experimental evidence for the negative charging predicted by theory [1,9]. Dimers are imaged as protruding features of nearly spherical shape. [Figure 2(e)]. Their bright appearance at positive bias indicates the presence of an empty energy level and a pronounced $d I / d V$ peak is indeed detected at 2.5-2.9 V [16]. The peak is assigned to the $p_{\perp}$ orbital, which is supported by the good agreement between the calculated orbital symmetry and its round appearance in $d I / d V$ maps [Fig. 2(e)] [17]. Trimers are identified as elongated protrusions oriented along the [001] direction (Fig. 3). Two well-separated $d I / d V$ peaks are detected at +2.3 and $+3.0 \mathrm{~V}$, characterized by a single maximum and a two-lobe structure in their $d I / d V$ maps, respectively, [Fig. 2(f)] [17]. This signature is compatible with the calculated trimer LDOS, revealing two $p_{\perp}$-derived states of similar node structure and energy. In general, no occupied states are observed for the ultrasmall Au clusters.

For aggregates containing more than three atoms, DFT predicts the formation of $\mathrm{Au}$ chains that run atop an $\mathrm{Al}$ row of the NiAl (Fig. 3). Their electronic structure is described by a series of QWS, evolving from the $s-d_{z^{2}}$ orbital of the monomer. The QWS resemble the eigenstates of a 1D box potential and exhibit a number of maxima in the axial electron density that is equal to the quantum number $n$ of the states $[18,19]$. The calculated energies $E_{n}$ of QWS for $\mathrm{Au}_{3}-\mathrm{Au}_{7}$ chains follow a parabolic dispersion $E_{n}=E_{0}+$ $\frac{\hbar^{2}}{2 m_{\text {eff }}}\left(\frac{n \pi}{L}\right)^{2}$, using the measured chain length $L$ (Fig. 3), an effective electron mass of $0.8 m_{e}$ and an onset energy of $-3.0 \mathrm{eV}$. Another set of empty QWS develops from the $p_{\perp}$ orbitals and starts at $1.6 \mathrm{eV}$ above $E_{F}$. From the lobe number of the highest occupied QWS (HOMO), multiplied by two to account for the spin degeneracy, the maximum number of electrons in the QWS is determined for each chain (Table I).

STM topographies of the system show indeed chainlike protrusions with [001] orientation (Fig. 3) [10]. Spectroscopy of their occupied states reveals a $d I / d V$ peak, which is assigned to the HOMO and characterized by a number of lobes that increases with chain length. For longer chains, also the HOMO-1 is detected with a lobe number reduced by one with respect to the HOMO (Fig. 3, bottom). Based on the DFT results, the smallest aggregate with resolved $\mathrm{HOMO}$ is assigned to an $\mathrm{Au}_{4}$ chain. It carries six $s$ electrons, as the HOMO is well below $E_{F}$ and shows three maxima in experiment and theory $(n=3)$. The $\mathrm{Au}$ atoms donate four electrons to the QWS, while the remaining two are introduced from the $\mathrm{NiAl}$ (Table I). As for the $\mathrm{Au}_{3}$, only the outer chain atoms bind to the oxide surface and are responsible for the charge transfer [Fig. 1(c)]. The experimental HOMOs of the two longer chains display four and five lobes. Taking also their topographic length into account, best matching is achieved with five- and seven-atom chains. In both cases, three $\mathrm{Al}_{\text {top }}-\mathrm{O}_{\text {inter }}$ bonds



FIG. 3 (color online). Experimental and calculated HOMO shapes, topographies and model structures for $\mathrm{Au}_{3}, \mathrm{Au}_{4}, \mathrm{Au}_{5}$ and $\mathrm{Au}_{7}$ chains. Images are $5.0 \times 5.0 \mathrm{~nm}^{2}$ in size. For the $\mathrm{Au}_{7}$ chain, the HOMO-1 is shown in addition. Measured chain lengths are $9 \AA, 12 \AA, 15 \AA$, and $22 \AA$; calculated distances between first and last chain atom amount to 5.3, 7.8, 10.5, and $15.5 \AA$. To compare theoretical to experimental lengths, $2-3 \AA$ should be added to both chain sides to account for the diffusivity of the $1 \mathrm{D}$ orbitals. 


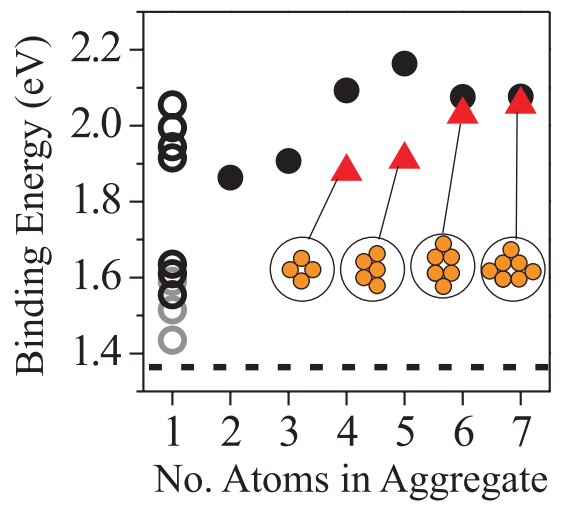

FIG. 4 (color online). Calculated binding energies for $\mathrm{Au}$ monomers in special four- and fivefold coordinated $\mathrm{Al}_{\text {top }}$ sites (black and gray open circles) as well as for atoms in linear chains (filled circles) and 2D islands shown in the insets (triangles). The broken line marks the average binding energy of all $26 \mathrm{Al}_{\text {top }}$ sites in the oxide unit cell.

are broken in the alumina; thus, three electrons are transferred into the QWS. This brings the total number of electrons to eight for the $\mathrm{Au}_{5}$ (five $\mathrm{Au}$ plus three transferelectrons) and ten for the $\mathrm{Au}_{7}$ chain $(7+3)$ (Table I). Also the $\mathrm{HOMO}$ of the $\mathrm{Au}_{6}$ chain would display five nodal planes. Because of the uneven number of QWS electrons $(6+3)$ in this case, the $\mathrm{Au}_{6}$ HOMO is only singly occupied (magnetic ground state) and located directly at $E_{F}$, which might explain its absence in the experimental records. In general, the number of QWS electrons and the magnetic structure of the ad-chains cannot be deduced from the number of chain atoms and are only explained when considering the charge transfer from the support.

The influence of the $\mathrm{NiAl}$ is also the driving force for the self-organization of Au atoms into linear ad-chains. The support strengthens the Au-alumina interaction at special $\mathrm{Al}_{\text {top }}$ sites by stabilizing the $\mathrm{O}_{\text {inter }}$ species below an $\mathrm{Au}$ via bond formation to an $\mathrm{Al}$ atom in the metal [Fig. 1(b)]. The linear arrangement of $\mathrm{Au}$ atoms now reflects the alignment of favorable $\mathrm{Al}_{\text {top }}$ sites above one and the same [001] oriented $\mathrm{Al}$ row. The formation of $2 \mathrm{D}$ islands, in contrast, requires the occupation of unfavorable sites above a $\mathrm{Ni}$ row, which is not balanced by the additional Au-Au bonds. The Au adsorption energies in 1D chains are therefore generally higher than in 2D aggregates for small atom numbers (Fig. 4). The chain growth comes to an end, because the misalignment of $6^{\circ}$ between $\mathrm{Al}$ rows in the $\mathrm{NiAl}$ and oxide $\mathrm{Al}_{\text {top }}$ rows eventually destroys the favorable binding constellation [10]. Following DFT calculations, the stability transition between 1D and 2D structures occurs for 7-8 atoms per aggregate and $\mathrm{Au}_{7}$ chains are indeed the longest ones observed experimentally.

In conclusion, the assembly of Au adatoms into chains is induced by the linear arrangement of favorable adsorption sites, which enables a particularly strong binding to the alumina/NiAl support. This unusual interaction mecha- nism is owed to the electronegative character of $\mathrm{Au}$ and the resulting charge transfer through the oxide film and not expected for the respective bulk oxides [15].

J.S. and H. J.F. acknowledge support from the Cluster of Excellence "Unifying Concepts in Catalysis" funded by the DFG as well as from the "Fond der Chemischen Industrie."

*Corresponding authors. nilius@fhi-berlin.mpg.de vgp@chemie.hu-berlin.de

†Present address: Department of Physics and Astronomy, University College London, Gower Street, London, United Kingdom.

[1] M. Sterrer et al., Phys. Rev. Lett. 98, 096107 (2007).

[2] G. Pacchioni, L. Giordano, and M. Baistrocchi, Phys. Rev. Lett. 94, 226104 (2005); L. Giordano, F. Cinquini, and G. Pacchioni, Phys. Rev. B 73, 045414 (2006).

[3] B. M. Sterrer, T. Risse, M. Heyde, H.-P. Rust, and H.-J. Freund, Phys. Rev. Lett. 98, 206103 (2007).

[4] M. S. Chen and D. W. Goodman, Science 306, 252 (2004); H. Yoon et al., Science 307, 403 (2005).

[5] H. J. Freund, Surf. Sci. 601, 1438 (2007); M. Bäumer and H.-J. Freund, Prog. Surf. Sci. 61, 127 (1999).

[6] R. D. Diehl and R. McGrath, Surf. Sci. Rep. 23, 43 (1996).

[7] A. S. Worz, U. Heiz, F. Cinquini, and G. Pacchioni, J. Phys. Chem. B 109, 18418 (2005).

[8] M. Frank and M. Bäumer, Phys. Chem. Chem. Phys. 2, 3723 (2000).

[9] J. Repp, G. Meyer, F. E. Olsson, and M. Persson, Science 305, 493 (2004).

[10] M. Kulawik, N. Nilius, and H.-J. Freund, Phys. Rev. Lett. 96, 036103 (2006).

[11] H.-P. Rust, J. Buisset, E. K. Schweizer, and L. Cramer, Rev. Sci. Instrum. 68, 129 (1997).

[12] J. P. Perdew et al., Phys. Rev. B 46, 6671 (1992); G. Kresse and J. Furthmüller, Comput. Mater. Sci. 6, 15 (1996).

[13] G. Kresse, M. Schmid, E. Napetschnig, M. Shishkin, L. Kohler, and P. Varga, Science 308, 1440 (2005); M. Schmid et al., Phys. Rev. Lett. 97, 046101 (2006).

[14] The role of $\mathrm{NiAl}$ in the Au binding was initially discovered by M. Shishkin and G. Kresse (private communication).

[15] P. Pyykko, Angew. Chem., Int. Ed. 43, 4412 (2004); Inorg. Chim. Acta 358, 4113 (2005).

[16] The scatter of peak energies reflects the presence of different dimer configurations, which are indistinguishable in the STM images.

[17] At high positive bias, the $d I / d V$ signal above an $\mathrm{Au}$ aggregate is lower than above the alumina film due to the enlarged tip distance from the NiAl.

[18] N. Nilius, T.M. Wallis, and W. Ho, Science 297, 1853 (2002); M. Persson, Phys. Rev. B 70, 205420 (2004).

[19] S. Fölsch, P. Hyldgaard, R. Koch, and K. H. Ploog, Phys. Rev. Lett. 92, 056803 (2004). 